\title{
Local-to-global frames and applications to dynamical sampling problem
}
A. Aldroubi*
C. Cabrelli ${ }^{\dagger}$
U. Molter
A. Petrosyan ${ }^{\S}$

\begin{abstract}
In this paper we consider systems of vectors in a Hilbert space $\mathcal{H}$ of the form $\left\{g_{j k}: j \in\right.$ $J, k \in K\} \subset \mathcal{H}$ where $J$ and $K$ are countable sets of indices. We find conditions under which the local reconstruction properties of such a system extend to global stable recovery properties on the whole space. As a particular case, we obtain new local-to-global results for systems of type $\left\{A^{n} g\right\}_{g \in \mathcal{G}, 0 \leq n \leq L}$ arising in the dynamical sampling problem.
\end{abstract}

\section{Introduction}

The notion of frame was originally introduced by Duffin and Schaffer [27] in the context of nonharmonic analysis, in particular exponential systems of the form $\left\{e^{2 \pi i \lambda_{i} x}\right\}_{i \in I}$ where $\left\{\lambda_{i}\right\}_{i \in I} \subset \mathbb{R}$ were considered. However, the notion found wide applications in signal processing research and it has attracted a great deal of interest from the mathematics as well as the engineering communities during the last several decades.

Definition 1.1. A system of vectors $\mathcal{F}=\left\{f_{i}\right\}_{i \in I} \subset \mathcal{H}$ is said to be a frame for the Hilbert space $\mathcal{H}$ if there exist two constants $\alpha, \beta>0$ such that

$$
\alpha\|f\|^{2} \leq \sum_{i \in I}\left|\left\langle f, f_{i}\right\rangle\right|^{2} \leq \beta\|f\|^{2},
$$

for all $f \in \mathcal{H}$. If only the upper bound holds, then the system is called a Bessel system.

Frames are a generalization of bases. In particular, if $\left\{f_{i}\right\}_{i \in I}$ is a frame for $\mathcal{H}$, then any vector $f \in \mathcal{H}$ has the representation $f=\sum_{i \in I}\left\langle f, f_{i}\right\rangle \tilde{f}_{i}$ where $\left\{\tilde{f}_{i}\right\}_{i \in I}$ is another frame called the canonial dual frame. However, unlike bases, the vectors in a frame $\left\{f_{i}\right\}_{i \in I}$ may have linear dependencies thus resulting in a redundant representation (see [21] for more details). If the frame system is not a basis, then a frame $\left\{f_{i}\right\}_{i \in I}$ has many dual frames other than the canonical dual frame.

In signal processing models, often the system $\mathcal{F}$ consists of functions, defined on the domain $\Gamma=\mathbb{Z}$ or $\mathbb{R}$. The functions $f_{i}$ are usually localized. By that we mean that every point in the domain is contained in the supports of only finitely many functions from the system. This may happen, for example, when $\mathcal{F}$ is generated by the shifts of a finite number of compactly supported functions. In this setting, we may be able to divide the domain into smaller patches such that every function $f$ can be recovered on each patch, from inner products with those functions from $\mathcal{F}$ whose support overlap with that patch, i.e., $f$ can be recovered from local information. For that reason, we consider systems $\mathcal{F}$ re-indexed to have the form $\left\{g_{j k}: j \in J, k \in K\right\} \subset \mathcal{H}$ where the index $j \in J$ corresponds to the

\footnotetext{
*Department of Mathematics, Vanderbilt University, Nashville TN, USA, email: akram.aldroubi@vanderbilt.edu

†Departamento de Matemática, Universidad de Buenos Aires, Argentina, email: cabrelli@dm.uba.ar

‡Departamento de Matemática, Universidad de Buenos Aires, Argentina, email: umolter@dm.uba.ar

$\S$ Computational and Applied Mathematics Group, Oak Ridge National Laboratory, Oak Ridge TN, USA, email: petrosyana@ornl.gov
} 
patch $j$ and the index in $k \in K$ corresponds to indexing within each patch. The index sets $I, J, K$ will always be at most countable. The main interest in this note is to find conditions that guarantee stable global reconstruction of any function on the whole domain $\Gamma$, given that the system has local recovery properties. In that regard, among other results, we prove the following theorem.

Theorem. Let $\mathcal{H}$ be a Hilbert space and $\left\{P_{j}\right\}_{j \in \mathbb{N}}$ be projectors acting on $\mathcal{H}$ satisfying $P_{r} P_{j}=0$ for $r \neq j$, and also $\sum_{j} P_{j}=\mathbb{1}$. Assume that $\left\{P_{j} g_{j k}: k \in K\right\}$ form a frame for $P_{j} \mathcal{H}$ with uniform frame bounds $\alpha, \beta$, and $\left\|P_{r} g_{j k}\right\| \leq\left|c_{k}(j-r)\right|$ with $c_{k} \in \ell^{1}(\mathbb{Z})$ for all $k \in K$. If $\sum_{k \in K}\left\|c_{k}\right\|_{1}<\alpha$, then $\left\{g_{j k}\right\}_{j \in J, k \in K}$ is a frame for $\mathcal{H}$ with frame bounds $\alpha-\sum_{k \in K}\left\|c_{k}\right\|_{1}$ and $\beta+\sum_{k \in K}\left\|c_{k}\right\|_{1}$.

As a main application of our results, we study the Dynamical Sampling (DS) problem where one wants to recover an unknown signal $f$ from spatial-temporal samples $\left\{\left\langle f, A^{n} g\right\rangle\right\}_{g \in \mathcal{G}, 0 \leq n \leq L}$ where $L<\infty$, and $\mathcal{G}$ is a collection of functions defined in $\Gamma$. Here $\Gamma$ is either $\mathbb{R}$ (continuous case) or $\mathbb{Z}$ (discrete case), and $A$ is an operator acting on $L^{2}(\Gamma)$. This leads to the study of frame properties of the iterative system

$$
\left\{A^{n} g\right\}_{g \in \mathcal{G}, 0 \leq n \leq L} .
$$

The dynamical sampling problem was introduced in [6], and it is an active area of research in the applied harmonic analysis community, with a range of potential fields of applications [8]. Early results in DS considered convolution operators on the spaces $l^{2}\left(\mathbb{Z}_{d}\right), l^{2}(\mathbb{Z})$ and $L^{2}(\mathbb{R})$ with the samples taken on a sparse uniform grid [6,7]. In $[10,33]$, authors allowed the operator $A$ to be unknown too. For an operator theoretic approach to the dynamical sampling problem see $[3,5,9,12,32]$. The case when $f \in l^{2}(\mathbb{N}), A=B^{-1} D B$ and $D=\sum_{j} \lambda_{j} P_{j}$ is an infinite diagonal matrix can be found in [5]. For general bounded normal operators see $[12,16,15]$. The authors in $[22,23,24,25,26]$ consider general frames and investigate conditions under which they can be represented as iterated systems arising in dynamical sampling. In [11] authors study the problem of phaseless recovery from dynamical samples. The scalability properties of the system arising in DS is investigated in [2].

Our main results in this paper are proved for general class of systems and the results are applied to dynamical sampling. In particular, we consider dynamical systems where the system $\mathcal{G}$ is localized in space (e.g. when it is generated by the shift of a single compactly supported function), and $A$ is a compactly supported convolution operator. More specifically, we obtain the following theorem as corollary of the results in Section 2.

Theorem. Let a be a finite sequence, and consider the convolution operator $A f=a * f$ for $f \in \ell^{2}(\mathbb{Z})$. Let $I=\{-N, \ldots, N\}$ and $I_{j}=I+N_{j} \subset \mathbb{Z}$ be such that $\cup_{j} I_{j}=\mathbb{Z}$, and $I_{j} \cap I_{k}=\emptyset$ for $|j-k|>M$. Assume that $G_{0}=\left\{\chi_{I_{j}} a^{n}(\cdot-l): l \in \Omega \subset I\right\}$ is a frame for $\ell^{2}(I)\left(\chi_{I_{j}}\right.$ is the characteristic function of $\left.I_{j}\right)$, and let $S_{k}$ be the shift operator by the integer $k$. Then $\cup_{j} S_{N_{j}} G_{0}$ is a frame for $\ell^{2}(\mathbb{N})$.

The paper is organized as follows. In Section 2 we prove our main results concerning general systems with local-to-global indexing. In Section 3, we apply the results from Section 2 to iterative system of vectors arising from dynamical sampling problem.

\section{Main results for general systems}

In this section we prove our main theorems concerning local-to-global frame properties of the systems $\left\{g_{j k}: j \in J, k \in K\right\} \subset \mathcal{H}$ where $J$ and $K$ are countable (finite or infinite). Here $g_{j, k}$ are some vectors, not necessarily functions on $\mathbb{R}$ or $\mathbb{Z}$. The local patches are replaced with projection operators $\left\{P_{j}\right\}_{j \in J}$ or more generally with operators $\left\{T_{j}\right\}_{j \in J} \subset B(\mathcal{H})$. Our first proposition gives a way to construct a global frame from local frames. This is a generalization of Theorem 5.3 from [4].

Proposition 2.1. If $\left\{T_{j}\right\}_{j \in J} \subset B(\mathcal{H}), T_{j}$ has close range for each $j, A\|f\|^{2} \leq \sum_{j}\left\|T_{j} f\right\|^{2} \leq B\|f\|^{2}$ for all $f \in \mathcal{H}$ for some $A, B>0$, and the set $\left\{T_{j} g_{j k}: k \in K\right\}$ forms a frame for $T_{j}(\mathcal{H})$ with uniform frame bounds $\alpha, \beta$, then $\left\{T_{j}^{*} T_{j} g_{j k}: j \in J, k \in K\right\}$ is a frame for $\mathcal{H}$ with frame bounds $\alpha A, \beta B$. 
Conversely, if $\left\{T_{j} g_{j k}: k \in K\right\}$ forms a frame for $T_{j}(\mathcal{H})$ with uniform frame bounds $\alpha, \beta$, and $\left\{T_{j}^{*} T_{j} g_{j k}: j \in J, k \in K\right\}$ is a frame for $\mathcal{H}$ with frame bounds $\alpha A, \beta B$, then

$$
\frac{\alpha}{\beta} A\|f\|^{2} \leq \sum_{j}\left\|T_{j} f\right\|^{2} \leq \frac{\beta}{\alpha} B\|f\|^{2} \quad \text { for all } f \in \mathcal{H} .
$$

Proof. Let $f \in \mathcal{H}$. Then

$$
\alpha\left\|T_{j} f\right\|^{2} \leq \sum_{k \in K}\left|\left\langle T_{j} f, T_{j} g_{j k}\right\rangle\right|^{2}=\sum_{k \in K}\left|\left\langle f, T_{j}^{*} T_{j} g_{j k}\right\rangle\right|^{2} .
$$

Summing over $j \in J$ we get

$$
\alpha A\|f\|^{2} \leq \alpha \sum_{j \in J}\left\|T_{j} f\right\|^{2} \leq \sum_{j \in J} \sum_{k \in K}\left|\left\langle f, T_{j}^{*} T_{j} g_{j k}\right\rangle\right|^{2}
$$

which establishes the lower frame bound for the set $\left\{T_{j}^{*} T_{j} g_{j k}: j \in J, k \in K\right\}$. A similar calculation yields the upper bound.

To prove the second part, let $f \in \mathcal{H}$. Then

$$
\sum_{k \in K}\left|\left\langle f, T_{j}^{*} T_{j} g_{j k}\right\rangle\right|^{2}=\sum_{k \in K}\left|\left\langle T_{j} f, T_{j} g_{j k}\right\rangle\right|^{2} \leq \beta\left\|T_{j} f\right\|^{2} .
$$

Summing over $j \in J$, we get

$$
\alpha A\|f\|^{2} \leq \sum_{j \in J} \sum_{k \in K}\left|\left\langle f, T_{j}^{*} T_{j} g_{j k}\right\rangle\right|^{2} \leq \beta \sum_{j \in J}\left\|T_{j} f\right\|^{2} .
$$

Thus, $\frac{\alpha}{\beta} A\|f\|^{2} \leq \sum_{j \in J}\left\|T_{j} f\right\|^{2}$. Similar calculations yield the upper bound.

For the next proposition, we recall the definition of fusion frames (see $[17,18,19,31]$ and the references therein) and completeness for a system of orthogonal projectors.

Definition 2.2. A system of orthogonal projections $\left\{P_{j}\right\}_{j \in J}$ is said to be a fusion frame if there exists positive constants $m, M>0$ such that

$$
m\|f\|^{2} \leq \sum_{j}\left\|P_{j} f\right\|^{2} \leq M\|f\|^{2}, \quad f \in \mathcal{H} .
$$

Definition 2.3. A system of projections $\left\{P_{j}\right\}_{j \in \mathbb{N}}$ is said to be complete if $P_{j} f=0$ for all $j$ implies that $f=0$.

Using the fact that for orthogonal projector $P_{j}, P_{j}=P_{j}^{2}$ and $P_{j}=P_{j}^{*}$, Proposition 2.1 immediately yields the following corollary:

Proposition 2.4. If $\left\{P_{j}\right\}_{j \in J}$ is a fusion frame with bounds $A, B$, and the set $\left\{P_{j} g_{j k}: k \in K\right\}$ form a frame for $P_{j}(\mathcal{H})$ with uniform frame bounds $\alpha, \beta$, then $\left\{P_{j} g_{j k}: j \in J, k \in K\right\}$ is a frame for $\mathcal{H}$ with frame bounds $\alpha A, \beta B$.

Conversely, if $\left\{P_{j} g_{j k}: k \in K\right\}$ form a frame for $P_{j}(\mathcal{H})$ with uniform frame bounds $\alpha, \beta$, and $\left\{P_{j} g_{j k}: j \in J, k \in K\right\}$ is a frame for $\mathcal{H}$ with frame bounds $\alpha A, \beta B$, then $\left\{P_{j}\right\}_{j \in J}$ is a fusion frame with bounds $\frac{\alpha}{\beta} A$, and $\frac{\beta}{\alpha} B$.

Theorem 2.5. Assume that $\left\{P_{j}\right\}_{j \in \mathbb{N}}$ is such that $P_{j} P_{k}=P_{k} P_{j}$ for all $j, k \in \mathbb{N}$, and $P_{j} P_{k}=0$ for $|j-k| \geq M$ for some positive integer $M$. If $\left\{P_{j}\right\}_{j \in \mathbb{N}}$ is complete, then $\left\{P_{j}\right\}_{j \in \mathbb{N}}$ is a fusion frame. 
Proof. The set $\left\{P_{j}\right\}_{j \in \mathbb{N}}$ is the union of $M$ sets $\left\{P_{j}\right\}_{j \in \mathbb{N}}=\bigcup_{k=1}^{M}\left\{P_{k+M j}\right\}_{j \in \mathbb{N}}$. Using the fact that for each $k, \sum_{j \in \mathbb{N}} P_{k+M j}$ is an orthogonal projection, we get that for $f \in \mathcal{H}$

$$
\begin{aligned}
\left\|\sum_{j \in \mathbb{N}} P_{j} f\right\|^{2} & =\left\|\sum_{k=1}^{M} \sum_{j \in \mathbb{N}} P_{k+M j} f\right\|^{2} \\
& \leq \sum_{k=1}^{M}\left\|\sum_{j \in \mathbb{N}} P_{k+M j} f\right\|^{2} \\
& \leq M\|f\|^{2} .
\end{aligned}
$$

To prove the lower bound we proceed by induction. We first note that, because $P_{1}, P_{2}$ commute, $P_{1} P_{2}$ is an orthogonal projector on $P_{1}(\mathcal{H}) \cap P_{2}(\mathcal{H})$. Let $Q_{1}=P_{1}, Q_{2}=P_{2}-P_{2} Q_{1}$. Then, $Q_{2}^{*}=Q_{2}$, $Q_{2}^{2}=Q_{2}$, and $Q_{1} Q_{2}=0$. Moreover, since $P_{1} Q_{2}=0$, we get

$$
\left\|Q_{1} f\right\|^{2}+\left\|Q_{2} f\right\|^{2}=\left\|P_{1} f\right\|^{2}+\left\|\left(P_{2}-P_{2} P_{1}\right) f\right\|^{2}=\left\|P_{1} f\right\|^{2}+\left\|\left(\mathbb{1}-P_{1}\right) P_{2} f\right\|^{2} \leq\left\|P_{1} f\right\|^{2}+\left\|P_{2} f\right\|^{2} .
$$

For $n>2$, let $Q_{n}=P_{n}-P_{n} \sum_{k=1}^{n-1} Q_{k}$. Then, using the fact that $R=\sum_{k=1}^{n-1} Q_{k}$ commutes with $P_{n}$, it is not difficult to verify that $Q_{n}^{*}=Q_{n}$, and $Q_{n}^{2}=Q_{n}$ and that $Q_{n} R=0$. Assume that $Q_{j} Q_{k}=0$ for $j, k<n$. Then, applying $Q_{l}$ to both side of the equation $Q_{n} R=0$, we get that $Q_{n} Q_{l}=0$ for all $l<n$. Thus, $Q_{j} Q_{k}=0$ for $j \neq k$. Using induction again, we have

$$
\|R f\|^{2}+\left\|Q_{n} f\right\|^{2}=\sum_{j=1}^{n-1}\left\|Q_{j} f\right\|^{2}+\left\|(\mathbb{1}-R) P_{n} f\right\|^{2} \leq \sum_{j=1}^{n-1}\left\|P_{j} f\right\|^{2}+\left\|P_{n} f\right\|^{2} .
$$

Hence

$$
\sum_{j \in \mathbb{N}}\left\|Q_{j} f\right\|^{2} \leq \sum_{j \in \mathbb{N}}\left\|P_{j} f\right\|^{2} .
$$

Since $Q_{1}=P_{1}, Q_{n}=P_{n}-P_{n} R$ for $n \geq 2$, we get that $\left\{Q_{j}\right\}$ is also complete whenever $P_{j}$ is complete. Hence,

$$
\|f\|^{2}=\sum_{j \in \mathbb{N}}\left\|Q_{j} f\right\|^{2} \leq \sum_{j \in \mathbb{N}}\left\|P_{j} f\right\|^{2}
$$

Combining Proposition 2.4 with Theorem 2.5, we obtain the following corollary.

Corollary 2.6. Assume that $\left\{P_{j}\right\}_{j \mathbb{N}}$ is complete and is such that $P_{j} P_{k}=P_{k} P_{j}$ for all $j, k \in \mathbb{N}$, and $P_{j} P_{k}=0$ for $|j-k| \geq M$ for some positive integer $M$. Furthermore assume that $\left\{P_{j} g_{j k}: k \in K\right\}$ from a frame for $P_{j} \mathcal{H}$ with uniform frame bounds $\alpha, \beta$ and that $P_{j} g_{j k}=g_{j k}$ for all $j, k$. Then, the set $\left\{g_{j k}\right\}_{j \in J, k \in K}$ is a frame for $\mathcal{H}$.

In the previous corollary the assumption that $P_{j} g_{j k}=g_{j k}$ is rather strong and does not always happen in practice. The next theorem offers a way to overcome this assumption.

Theorem 2.7. Let $\left\{P_{j}\right\}_{j \mathbb{N}}$ be orthogonal projectors satisfying $P_{r} P_{j}=0$ for $r \neq j$, and $\sum_{j} P_{j}=\mathbb{1}$. Assume that $\left\{P_{j} g_{j k}: k \in K\right\}$ form a frame for $P_{j} \mathcal{H}$ with uniform frame bounds $\alpha, \beta$, and $\left\|P_{r} g_{j k}\right\| \leq$ $\left|c_{k}(j-r)\right|$ with $c_{k} \in \ell^{1}(\mathbb{Z})$. If $\sum_{k=1}^{K}\left\|c_{k}\right\|_{1}<\alpha$, then $\left\{g_{j k}\right\}_{j \in J, k \in K}$ is a frame for $\mathcal{H}$ with frame bounds $\alpha-\sum_{k=1}^{K}\left\|c_{k}\right\|_{1}$ and $\beta+\sum_{k=1}^{K}\left\|c_{k}\right\|_{1}$. 
Proof. For the lower bound we have

$$
\begin{aligned}
\left(\sum_{j, k}\left|\left\langle f, g_{j k}\right\rangle\right|^{2}\right)^{1 / 2} & =\left(\sum_{j, k}\left|\left\langle\sum_{l} P_{l} f, g_{j k}\right\rangle\right|^{2}\right)^{1 / 2} \\
& =\left(\sum_{j, k}\left|\left\langle P_{j} f, g_{j k}\right\rangle+\left\langle\sum_{l \neq j} P_{l} f, g_{j k}\right\rangle\right|^{2}\right)^{1 / 2} \\
& \geq\left(\sum_{j, k}\left|\left\langle P_{j} f, g_{j k}\right\rangle\right|^{2}\right)^{1 / 2}-\left(\sum_{j, k}\left|\left\langle\sum_{l \neq j} P_{l} f, g_{j k}\right\rangle\right|^{2}\right)^{1 / 2} \\
& \geq\left(\alpha \sum_{j}\left\|P_{j} f\right\|^{2}\right)^{1 / 2}-\left(\sum_{j, k} \mid \sum_{l \neq j}\left\|P_{l} f\right\| c_{k}(l-j) \|^{2}\right)^{1 / 2} \\
& =\alpha^{1 / 2}\|f\|-\left(\sum_{j, k}\left|\sum_{l \neq j}\left\|P_{l} f\right\|\right| c_{k}(l-j) \|^{2}\right)^{1 / 2} \\
& \geq \alpha^{1 / 2}\|f\|-\left(\sum_{k} \sum_{l}\left\|P_{l} f\right\|^{2}\left\|c_{k}\right\|_{1}^{2}\right)^{1 / 2} \\
& =\alpha^{1 / 2}\|f\|-\left(\sum_{k}\left\|c_{k}\right\|_{1}^{2}\right)^{1 / 2}\|f\| .
\end{aligned}
$$

A similar calculation gives the upper bound.

\section{Application to DS}

In this section we apply our results from Section 2 to the dynamical sampling setting. As a direct corollary of the Theorem 2.7, we get the following theorem.

Theorem 3.1. Let $a \in \ell_{1}(\mathbb{Z})$ be such that for a set $\Omega \subset I=\{0, \ldots, N\}$, the system $\left\{\left.a^{k} * \delta_{i}\right|_{I}: i \in\right.$ $\Omega, k=0, \ldots, K\}$ is a frame in the space $\ell^{2}(I)$ with frame bounds $\alpha$ and $\beta$ where $a^{k}$ denotes the $k$ times convolution of a. Let $I_{j}=I-j N$ and $\Omega_{j}=\Omega-j N$. If

$$
\gamma=\sum_{i \in \Omega} \sum_{k=0, \ldots, K} \sum_{j \in \mathbb{Z} \backslash\{0\}}\left\|\left.a^{k} * \delta_{i}\right|_{I_{j}}\right\|_{\ell^{2}\left(I_{j}\right)}<\alpha
$$

then the system $\left\{a^{k} * \delta_{i}: i \in \cup_{j \in \mathbb{Z}} \Omega_{j}, k=0, \ldots, K\right\}$ is a frame for $\ell^{2}(\mathbb{Z})$ with frame bounds $\alpha-\gamma$ and $\beta+\gamma$.

To insure the assumptions in the above theorem are realistic, below we construct an example where they actually hold.

Example 1. Let $I=\{0,1,2\}, \Omega=\{0\}$ and

$$
a(i)= \begin{cases}1, & i=0 \\ \tau, & i=1 \\ \tau^{2}, & i=2 \\ 0, & \text { otherwise }\end{cases}
$$


where $\tau>0$. Then notice that

$$
a * a(i)= \begin{cases}1, & i=0 \\ 2 \tau, & i=1 \\ 3 \tau^{2}, & i=2 \\ 2 \tau^{3}, & i=3 \\ \tau^{4}, & i=4 \\ 0, & \text { otherwise }\end{cases}
$$

Hence

$$
\left\{\left.a^{k} * \delta_{0}\right|_{I}: k=0,1,2\right\}=\left\{(1,0,0),\left(1, \tau, \tau^{2}\right),\left(1,2 \tau, 3 \tau^{2}\right)\right\}
$$

which are linearly independent for $\tau>0$ since for the matrix

$$
\Phi=\left(\begin{array}{ccc}
1 & 0 & 0 \\
1 & \tau & \tau^{2} \\
1 & 2 \tau & 3 \tau^{2}
\end{array}\right)
$$

$\operatorname{det}(\Phi)=5 \tau^{3}$ and hence are a basis in $\ell^{2}(I)$.

The quantity $\gamma$ in Theorem 3.1 is equal to $2 \tau^{3}+\tau^{4}$. For sufficiently small values of $\tau$, it can be checked that the lower frame bound of the system, which is the smallest singular value of $\Phi$ is larger than $\gamma$ and thus the conditions of Theorem 3.1 are satisfied. For the value of $\tau=0.1$, the lower frame bound is approximately 0.0040 whereas $\gamma=0.0021$.

The next two propositions concern frames from dynamical sampling from diagonal and convolutional operators, and their proofs follow from Corollary 2.6.

Proposition 3.2. Let $D$ be an infinite diagonal matrix acting on $\ell^{2}(\mathbb{N})$. Assume that $D \in B\left(\ell^{2}\right)$. Let $\left\{I_{j}\right\}_{j \in \mathbb{N}} \subset \mathbb{N}$ be such that, $\left|I_{j}\right| \leq L, \cup_{j} I_{j}=\mathbb{N}$, and $I_{j} \cap I_{k}=\emptyset$ for $|j-k|>M$. Let $D_{j}$ be the submatrix of $D$ indexed by $I_{j}$, and let $G_{j} \subset \ell^{2}(\mathbb{N})$ be a set vectors such that, for any $g \in G_{j}$, $\operatorname{supp} g \subset I_{j}$ and $\left\{D_{j}^{n} g\right\}_{g \in G_{j}, 0 \leq n<L}$ is a frame for $\ell^{2}\left(I_{j}\right)$ with frame bounds $\alpha, \beta$ independent of $j$. Then the set $\left\{D^{n} g: 0 \leq n<L, g \in \cup_{j \in \mathbb{N}} G_{j}\right\}$ is a frame for $\ell^{2}(\mathbb{N})$.

As a specific instance let us consider the next two examples.

Example 2. Assume that the entries of the diagonal matrix $D$ are periodic on the diagonal, i.e., $D_{i i}=f(i)$ where $f(i+p)=f(i)$ for $i \in \mathbb{N}$. This case reduces, each submatrix $D_{j}$ of $D$ in the proposition to be one of finitely many possible matrices. For each of these matrices, we construct corresponding $G_{j}$. Since the construction uses finitely many matrices and finitely many associated vectors, we can find frame bounds $\alpha, \beta$ that are independent of $j$ and the conditions in Proposition 3.2 will be satisfied.

The next proposition concerns convolutional operators which covers a large class of operators of practical interest like the diffusion operator on $\ell^{2}(\mathbb{Z})$.

Proposition 3.3. Let a be a finite sequence, and consider the convolution operator $A f=a * f$ for $f \in \ell^{2}(\mathbb{Z})$. Let $I=\{-N, \ldots, N\}, \Omega \subset I$, and $I_{j}=I+N_{j} \subset \mathbb{Z}$ be such that $\cup_{j} I_{j}=\mathbb{Z}$, and $I_{j} \cap I_{k}=\emptyset$ for $|j-k|>M$. Assume that $G_{0}=\left\{\chi_{I_{j}} a^{n}(\cdot-l): l \in \Omega\right\}$ is a frame for $\ell^{2}(I)\left(\chi_{I_{j}}\right.$ is the characteristic function of $\left.I_{j}\right)$, and let $S_{k}$ be the shift operator by the integer $k$. Then $\cup_{j} S_{N_{j}} G_{0}$ is a frame for $\ell^{2}(\mathbb{N})$.

\section{Acknowledgments}

The research of A. Aldroubi is supported in part by NSF grant DMS- 1322099. C. Cabrelli and U. Molter were partially supported by grants: UBACyT 20020170100430BA, PICT 2014-1480 (ANPCyT) and CONICET PIP 11220110101018. A. Petrosyan acknowledges support by the Oak Ridge National Laboratory, which is operated by UT-Battelle, LLC., for the U.S. Department of Energy under Contract DE-AC05-00OR22725. 


\section{References}

[1] R. Aceska, J-L Bouchot, and Sh. Li. Fusion frames and distributed sparsity. In Frames and harmonic analysis, volume 706 of Contemp. Math., pages 47-61, Amer. Math. Soc., Providence, RI, 2018.

[2] R. Aceska and Y. Kim. Scalability of frames generated by dynamical operators. Frontiers in Applied Mathematics and Statistics, 3(22), 2017.

[3] A. Aldroubi, C. Cabrelli, A.F. Cakmak, U. Molter, and A. Petrosyan. Iterative actions of normal operators. Journal of Functional Analysis, 272(3):1121-1146, 2017.

[4] A. Aldroubi, C. Cabrelli, and U. Molter. Wavelets on irregular grids with arbitrary dilations matrices and frame atoms for $L^{2}\left(\mathbb{R}^{d}\right)$. Applied and Computational Harmonic Analysis, 17,119$140,2004$.

[5] A. Aldroubi, C. Cabrelli, U. Molter, and S. Tang. Dynamical Sampling. Appl. Comput. Harmon. Anal., 42(3), 378-401, 2017

[6] A. Aldroubi, J. Davis, and I. Krishtal. Dynamical sampling: time-space trade-off. Appl. Comput. Harmon. Anal., 34(3):495-503, 2013.

[7] A. Aldroubi, J. Davis, and I. Krishtal. Exact reconstruction of signals in evolutionary systems via spatiotemporal trade-off. Journal of Fourier Analysis and Applications, 21(1),11-31, 2015.

[8] A. Aldroubi, L. Huang, I. Krishtal, R.R. Lederman, A. Ledeczi, and P. Volgyesi. On noise and unknown evolution operators in dynamical sampling. arXiv preprint arXiv:1807.10866, 2018.

[9] A. Aldroubi, L. Huang, and A. Petrosyan. Frames induced by the action of continuous powers of an operator. arXiv preprint arXiv:1801.10103, 2018.

[10] A. Aldroubi and I. Krishtal. Krylov subspace methods in dynamical sampling. ArXiv e-prints, December 2014.

[11] A. Aldroubi and S. Krishtal, I .and Tang. Phaseless reconstruction from space-time samples. Applied and Computational Harmonic Analysis, 2018.

[12] A. Aldroubi and A. Petrosyan. Dynamical sampling and systems from iterative actions of operators. In Frames and Other Bases in Abstract and Function Spaces, pages 15-26. Springer, 2017.

[13] A. Alijani. Non-orthogonal fusion frames in Hilbert $C^{*}$-modules. Int. J. Wavelets Multiresolut. Inf. Process., 16(3):1850015, 10, 2018.

[14] B.G. Bodmann and J.I. Haas. Maximal orthoplectic fusion frames from mutually unbiased bases and block designs. Proc. Amer. Math. Soc., 146(6):2601-2616, 2018.

[15] C. Cabrelli, U. Molter, V. Paternostro, and F. Philipp. Finite sensor dynamical sampling. In Sampling Theory and Applications (SampTA), 2017 International Conference on, pages 50-54. IEEE, 2017.

[16] C. Cabrelli, U. Molter, V. Paternostro, and F. Philipp. Dynamical sampling on finite index sets. Journal D'Analyse Mathematique, in press., 2018.

[17] J. Cahill, P.G. Casazza, M. Ehler, and Sh. Li. Tight and random nonorthogonal fusion frames. In Trends in harmonic analysis and its applications, volume 650 of Contemp. Math., pages 23-36. Amer. Math. Soc., Providence, RI, 2015. 
[18] J. Cahill, P.G. Casazza, and S. Li. Non-orthogonal fusion frames and the sparsity of fusion frame operators. J. Fourier Anal. Appl., 18(2):287-308, 2012.

[19] P.G. Casazza, G. Kutyniok, and Sh. Li. Fusion frames and distributed processing. Appl. Comput. Harmon. Anal., 25(1):114-132, 2008.

[20] X. Chen and A.M. Powell. Randomized subspace actions and fusion frames. Constr. Approx., 43(1):103-134, 2016.

[21] O. Christensen. An Introduction to Frames and Riesz Bases (Applied and Numerical Harmonic Analysis). Birkhäuser, 2016.

[22] O. Christensen and M. Hasannasab. Operator representations of frames: boundedness, duality, and stability. Integral Equations and Operator Theory, 88(4), 483-499, 2017.

[23] O. Christensen and M. Hasannasab. Frame properties of systems arising via iterated actions of operators. Applied and Computational Harmonic Analysis, 46 (3), 664-673, 2018.

[24] O. Christensen, M. Hasannasab, and F. Philipp. Frame properties of operator orbits. arXiv preprint arXiv:1804.03438, 2018.

[25] O. Christensen, M. Hasannasab, and E. Rashidi. Dynamical sampling and frame representations with bounded operators. Journal of Mathematical Analysis and Applications, 463(2), 634-644, 2018.

[26] O. Christensen, M. Hasannasab, and D.T. Stoeva. Operator representations of sequences and dynamical sampling. arXiv preprint arXiv:1804.00077, 2018.

[27] R. J. Duffin and A. C. Schaeffer. A class of nonharmonic Fourier series. Trans. Amer. Math. Soc., 72:341-366, 1952.

[28] P. Găvruţa. On the duality of fusion frames. J. Math. Anal. Appl., 333(2):871-879, 2007.

[29] S.B. Heineken and P.M. Morillas. Oblique dual fusion frames. Numer. Funct. Anal. Optim., 39(7):800-824, 2018.

[30] J. Jiang and A.M. Powell. Sigma-delta quantization for fusion frames and distributed sensor networks. In Frames and other bases in abstract and function spaces, Appl. Numer. Harmon. Anal., pages 101-124. Birkhäuser/Springer, Cham, 2017.

[31] Sh. K. Kaushik and V. Kumar. On fusion frames in Banach spaces. Georgian Math. J., 18(1):121130, 2011.

[32] F. Philipp. Bessel orbits of normal operators. Journal of Mathematical Analysis and Applications, 448(2):767-785, 2017.

[33] S. Tang. System identification in dynamical sampling. Advances in Computational Mathematics, 43(3):555-580, 2017. 